\title{
SHORT COMMUNICATION \\ SELECTED CARDIOVASCULAR RISK MARKERS \\ IN VEGETARIANS AND SUBJECTS OF GENERAL \\ POPULATION
}

\author{
Martina Valachovičová1, Jana Príbojová1, Vladimír Urbánek', Lucia Birošová2 \\ 'Slovak Medical University, Bratislava, Slovak Republic \\ ${ }^{2}$ Department of Nutrition and Food Quality Assessment, Faculty of Chemical and Food Technology, Slovak University of Technology in Bratis- \\ lava, Bratislava, Slovak Republic
}

\section{SUMMARY}

Objective: Besides genetic factors there are environmental effects including nutritional habits which can influence the risk of age-related diseases. The aim of the study was to assess the age dependence of selected cardiovascular risk markers in two groups of subjects with different nutritional pattern.

Methods: In 470 long-term vegetarians and 478 subjects of general population the following indicators were measured: total cholesterol, $\mathrm{HDL}$-cholesterol, triacylglycerol, glucose, insulin concentrations, LDL-cholesterol, atherogenic index and insulin resistance IR(HOMA) were also calculated in studied subjects. Obtained data were evaluated according to age decades.

Results: Vegetarian vs. non-vegetarian concentrations of total cholesterol, LDL-cholesterol, insulin, and values of atherogenic index and $\operatorname{IR}(\mathrm{HOMA})$ were significantly reduced in all age decades. Vegetarian vs. non-vegetarian triacalglycerol concentrations were significantly reduced from 4th-7th decade. Vegetarian average decade values of all lipid parameters were in reference range. In non-vegetarian group, the risk average values of total cholesterol $(>5.2 \mathrm{mmol} / /)$ were found from 5 th -7 th decade, LDL-cholesterol $(>3.3 \mathrm{mmol} / /)$ in 7 th decade and atherogenic index $(>4)$ in 6 th-7th decade. In vegetarians vs. non-vegetarians were noted the average decade values for total cholesterol ranging from 4.01-4.59 vs. $4.48-5.67 \mathrm{mmol} / \mathrm{l}$, for triacylglycerols $1.00-1.33 \mathrm{vs.} 1.13-1.74 \mathrm{mmol} / \mathrm{l}$, for LDL-cholesterol $2.03-2.58 \mathrm{vs}$. $2.43-3.49 \mathrm{mmol} / \mathrm{l}$, for atherogenic index $2.72-3.31$ vs. $3.05-4.21$ and for $\mathrm{IR}(\mathrm{HOMA}) 0.99-1.15$ vs. $1.15-1.84$.

Conclusion: Our data show significantly reduced mean age decade values of lipid and non-lipid cardiovascular risk markers in all adult vegetarians. Smaller changes of markers between decades compared to non-vegetarians document a protective effect of vegetarian nutrition in prevention of cardiovascular disease.

Key words: total cholesterol, LDL-cholesterol, insulin resistance, age, nutrition

Address for correspondence: M. Valachovičová, Slovak Medical University, Limbová 12, 83303 Bratislava, Slovak Republic. E-mail: martina. valachovicova@szu.sk

https://doi.org/10.21101/cejph.a4529

\section{INTRODUCTION}

Ageing is a complex process that negatively affects the development of different systems. However, the rate of ageing in humans is not uniform due to genetic heterogeneity and the influence of various factors including nutrition (1). Age-related changes in body function or composition that could serve as a measure of biological age and predict the onset of age-related diseases or residual lifetime are termed biomarkers of ageing. Age is a major risk factor in many diseases and biomarkers could be subsequently used to identify individuals at high risk of developing age-associated diseases or disabilities (1-3).

Ageing belongs to main risk factors in development and incidence of cardiovascular diseases, which are very frequently diagnosed in older individuals $(4,5)$. The gradual prolongation of life requires adequate health care as well as healthy life style, appropriate physical activity and correct nutrition (6).
The main goal of this study was to assess the selected cardiovascular risk parameters - lipid parameters and markers of insulin resistance in two groups of subjects with different nutritional pattern in relation to age.

\section{MATERIALS AND METHODS}

Randomly selected group of 548 apparently healthy adult non-obese non-smoking subjects aged $21-70$ years were divided into two groups according to their nutritional habit. Vegetarian group consisted of 270 lacto-ovo-vegetarians (111 men, 159 women), who consumed plant food, dairy products and eggs. Non-vegetarian control group was represented by 278 persons of general population on traditional mixed diet (105 men, 173 women). Vegetarian group: average age 42.6 \pm 0.9 (SEM) years, BMI $22.6 \pm 0.2 \mathrm{~kg} / \mathrm{m}^{2}$, duration of vegetarianism $10.6 \pm 0.4$ years; 
non-vegetarian group: average age $42.3 \pm 0.8$ years, BMI $23.9 \pm 0.2$ $\mathrm{kg} / \mathrm{m}^{2}(\mathrm{p}<0.001)$. In terms of evaluation of age dependence of risk cardiovascular markers, all probands were divided into age groups by decades (Table 1). The volunteers were selected according to age, gender, nutritional habit, $\mathrm{BMI}<30 \mathrm{~kg} / \mathrm{m}^{2}$, no smoking and no supplementation subjects were selected from database of previous research university projects as well as from database of employees and students of Slovak Medical University and other universities in Bratislava. They had approximately similar physical activity (mental work, no active sports).

Venous blood was sampled after an overnight fasting by a standard procedure. Serum concentrations of total cholesterol, HDL-cholesterol, triacylglycerols, and glucose were examined using standard laboratory methods. Values of LDL-cholesterol were calculated in accordance with the Friedewald formula $($ LDL-cholesterol $=$ total cholesterol - triacylglycerols $/ 2.2-$ HDL-cholesterol). The atherogenic index was expressed as a ratio of total cholesterol and HDL-cholesterol. Serum concentrations of insulin were detected by electro-chemiluminescence immunoassay (Roche Elecsys Insulin Test). Insulin resistance values IR(HOMA) (HOMA - homeostasis model assessment) were calculated from fasting concentrations of insulin and glucose: $\operatorname{IR}($ HOMA) $=$ insulin $x$ glucose $/ 22.5$ (reference value $<2.2$ ). The intake of vitamins, mineral and trace elements only in natural form was allowed (no supplementation). The study was conducted in spring (April, May), the same number of vegetarian

Table 1. Group characteristics, lipid profile (concentrations of total cholesterol, triacyl glycerols, HDL-and LDL-cholesterol in $\mathrm{nmol} / 1$ ) and insulin resistance (glucose concentration in nmol/1 and insulin levels in $\mathrm{mU} / 1$ )

\begin{tabular}{|c|c|c|c|c|c|}
\hline Age decades & 3th & 4th & 5 th & 6th & 7th \\
\hline Age span (years) & $21-30$ & $31-10$ & $41-50$ & $51-60$ & $61-70$ \\
\hline \multicolumn{6}{|l|}{ Non-vegetarians } \\
\hline$N(m+w)$ & $113(46+67)$ & $102(44+58)$ & $95(41+54)$ & $92(40+52)$ & $76(34+42)$ \\
\hline Average age (years) & $25.6+0.3$ & $35.2+0.3$ & $45.0+0.4$ & $55.7+0.4$ & $64.9+0.5$ \\
\hline $\mathrm{BMI}\left(\mathrm{kg} / \mathrm{m}^{2}\right)$ & $22.3+0.3$ & $23.8+0.4$ & $24.3+0.4$ & $25.1+0.4$ & $25.3+0.3$ \\
\hline$>25$ & $12 \%$ & $27 \%$ & $29 \%$ & $35 \%$ & $36 \%$ \\
\hline$>30$ & 0 & 0 & 0 & 0 & 0 \\
\hline Duration of vegetarianism (years) & 0 & 0 & 0 & 0 & 0 \\
\hline Smokers & 0 & 0 & 0 & 0 & 0 \\
\hline Total cholesterol (mmol/1) & $4.48+0.08$ & $5.11+0.11$ & $5.24+0.11$ & $5.45+0.14$ & $5.67+0.13$ \\
\hline Triacylglycerols (mmol/1) & $1.13+0.06$ & $1.44+0.14$ & $1.61+0.12$ & $1.65+0.11$ & $1.74+0.12$ \\
\hline HDL-cholesterol (mmol/1) & $1.54+0.04$ & $1.48+0.05$ & $1.45+0.05$ & $1.41+0.04$ & $1.39+0.04$ \\
\hline LDL-cholesterol (mmol/1) & $2.43+0.07$ & $2.98+0.10$ & $3.07+0.11$ & $3.31+0.12$ & $3.49+0.14$ \\
\hline Atherogenic index & $3.05+0.09$ & $3.73+0.18$ & $3.84+0.16$ & $4.07+0.14$ & $4.21+0.18$ \\
\hline Glucose (mmol/1) & $4.33+0.04$ & $4.57+0.06$ & $4.72+0.08$ & $4.80+0.10$ & $4.85+0.13$ \\
\hline Insulin (mU/1) & $5.97+0.29$ & $6.50+0.06$ & $7.49+0.59$ & $7.98+0.36$ & $8.25+0.43$ \\
\hline $\mathrm{IR}(\mathrm{HOMA})$ & $1.15+0.06$ & $1.33+0.09$ & $1.54+0.13$ & $1.76+0.11$ & $1.84+0.13$ \\
\hline \multicolumn{6}{|l|}{ Vegetarians } \\
\hline$N(m+w)$ & $108(46+62)$ & $99(43+56)$ & $92(44+48)$ & $93(44+49)$ & $78(34+44)$ \\
\hline Average age (years) & $25.4+0.3$ & $35.2+0.4$ & $45.4+0.4$ & $55.4+0.5$ & $64.1+0.5$ \\
\hline $\mathrm{BMI}\left(\mathrm{kg} / \mathrm{m}^{2}\right)$ & $21.0+0.3^{\wedge}$ & $22.4+0.3^{\wedge}$ & $23.1+0.4^{\circ}$ & $23.5+0.3^{*}$ & $23.6+0.3^{*}$ \\
\hline$>25$ & $3 \%$ & $8 \%$ & $19 \%$ & $19 \%$ & $13 \%$ \\
\hline$>30$ & 0 & 0 & 0 & 0 & 0 \\
\hline Duration of vegetarianism (years) & $8.4+0.6$ & $10.9+0.7$ & $12.0+0.7$ & $12.9+0.9$ & $9.4+0.8$ \\
\hline Smokers & 0 & 0 & 0 & 0 & 0 \\
\hline Total cholesterol (mmol/1) & $4.01+0.06^{*}$ & $4.39+0.08^{*}$ & $4.50+0.10^{*}$ & $4.56+0.08^{*}$ & $4.59+0.08^{*}$ \\
\hline Triacylglycerols (mmol/1) & $1.00+0.05$ & $1.07+0.05^{\circ}$ & $1.27+0.08^{\circ}$ & $1.30+0.08^{\wedge}$ & $1.33+0.08^{\wedge}$ \\
\hline HDL-cholesterol (mmol/1) & $1.49+0.03$ & $1.51+0.04$ & $1.52+0.05$ & $1.50+0.05$ & $1.44+0.06$ \\
\hline LDL-cholesterol (mmol/1) & $2.03+0.05^{*}$ & $2.40+0.07^{*}$ & $2.48+0.10^{*}$ & $2.54+0.08^{*}$ & $2.58+0.07^{*}$ \\
\hline Atherogenic index & $2.72+0.06^{\wedge}$ & $3.06+0.10^{\wedge}$ & $3.17+0.14^{\wedge}$ & $3.27+0.13^{*}$ & $3.31+0.11^{*}$ \\
\hline Glucose (mmol/1) & $4.37+0.05$ & $4.47+0.06$ & $4.63+0.07$ & $4.61+0.07$ & $4.65+0.07$ \\
\hline Insulin (mU/1) & $5.09+0.29^{\circ}$ & $5.13+0.28^{\wedge}$ & $5.58+0.24^{\wedge}$ & $5.37+0.26^{*}$ & $5.36+0.24^{*}$ \\
\hline
\end{tabular}

The results are expressed as mean \pm SEM; significance levels: vegetarians vs. non-vegetarians; ${ }^{\circ} p<0.05,{ }^{\wedge} p<0.01,{ }^{*} p<0.001$ 
and non-vegetarian subjects divided according to age decades were analysed each week. The Student t-test and linear regression analysis were applied for final evaluation.

\section{RESULTS}

Compared to non-vegetarians, concentrations of total cholesterol, LDL-cholesterol and values of atherogenic index in vegetarians were significantly reduced in all age decades (Table 1, Fig. 1). Similarly, vegetarian triacylglycerol concentrations were significantly reduced from 4 th to 7 th decade. Vegetarian average decade values of all lipid parameters were in reference range. In non-vegetarian group, the risk average values of total cholesterol $(>5.2 \mathrm{mmol} / \mathrm{l})$ were found from 5 th to 7 th decade, LDL-cholesterol $(>3.3 \mathrm{mmol} / \mathrm{l})$ in 7 th decade and atherogenic index $(>4)$ in 6 th and 7 th decade. In healthy non-obese vegetarians vs. non-vegetarians were noted average decade values for total cholesterol ranging from $4.01-4.59$ vs. $4.48-5.67 \mathrm{mmol} / \mathrm{l}$, for triacylglycerols $1.00-1.33$ vs. $1.13-1.74 \mathrm{mmol} / \mathrm{l}$, for LDLcholesterol $2.03-2.58$ vs. $2.43-3.49 \mathrm{mmol} / 1$, and for atherogenic index $2.72-3.31$ vs. 3.05-4.21, respectively (Table1, Fig. 1).

Hyperinsulinemia and insulin resistance are critical components of the metabolic syndrome and the early manifestations of type 2 diabetes. Insulin resistance IR(HOMA) was based on assumption that healthy subjects with normal weight aged less than 35 years have the insulin resistance of 1 and $100 \%$ of beta cell function. Vegetarian vs. non-vegetarian IR(HOMA) and insulin values in presented study were significantly reduced in all age decades (Table 1). In healthy non-obese vegetarians vs. non-vegetarians were noted the average decade IR(HOMA) values $0.99-1.15$ vs. $1.15-1.84$, respectively. Linear equations of
IR(HOMA) and age show that age changes were less pronounced in vegetarians (linear equation in vegetarians: IR(HOMA) = 0.0041 age $+0.9017, \mathrm{r}=0.135$; linear equation in non-vegetarians: $\operatorname{IR}($ HOMA $)=0.0159$ age $+0.8990, r=0.284$ (Fig. 1Pra)

\section{DISCUSSION}

Nutrition knowledge from previous studies suggests that the consumption of saturated fatty acid (animal sources) is associated with hypercholesterolemia, while polyunsaturated fatty acids (plant oils, oil seeds, nuts, oil spreads) were reported to have a cholesterol lowering effect. Consumption of food high in dietary fiber (fruit, vegetables, legumes, whole grain products, nuts, seeds) is associated with a lower risk of cardiovascular disease because of the ability of soluble and insoluble fibers to reduce plasma total cholesterol, LDL cholesterol and glycemic index. The hypocholesterolemic effect of fibre is due to an increase in bile-acid binding and faecal sterol excretion. Fermentation of soluble fibre results in production of short-chain fatty acids that inhibit hepatic cholesterol synthesis. In addition, plant food contains also components able to reduce cardiovascular risk such as saponins (legumes), plant proteins, antioxidant nutrients, selenium, polyphenols, and flavonoids (7-12). Previously, the dietary recommendations to reduce cardiovascular risk were aimed at decreasing of total and saturated fatty acids intake from meat consumption. Actually, the effect of this factor only may not be sufficient. Various plant foods in nutritional pattern are necessary for favourable modification of lipid and lipoprotein profile $(13,14)$.

Similar findings were described by Richter et.al. (15). The authors investigated 10,550 subjects of general population $(3,816$ men, 6,734 women) aged 18-93 years compared with 417 veg-

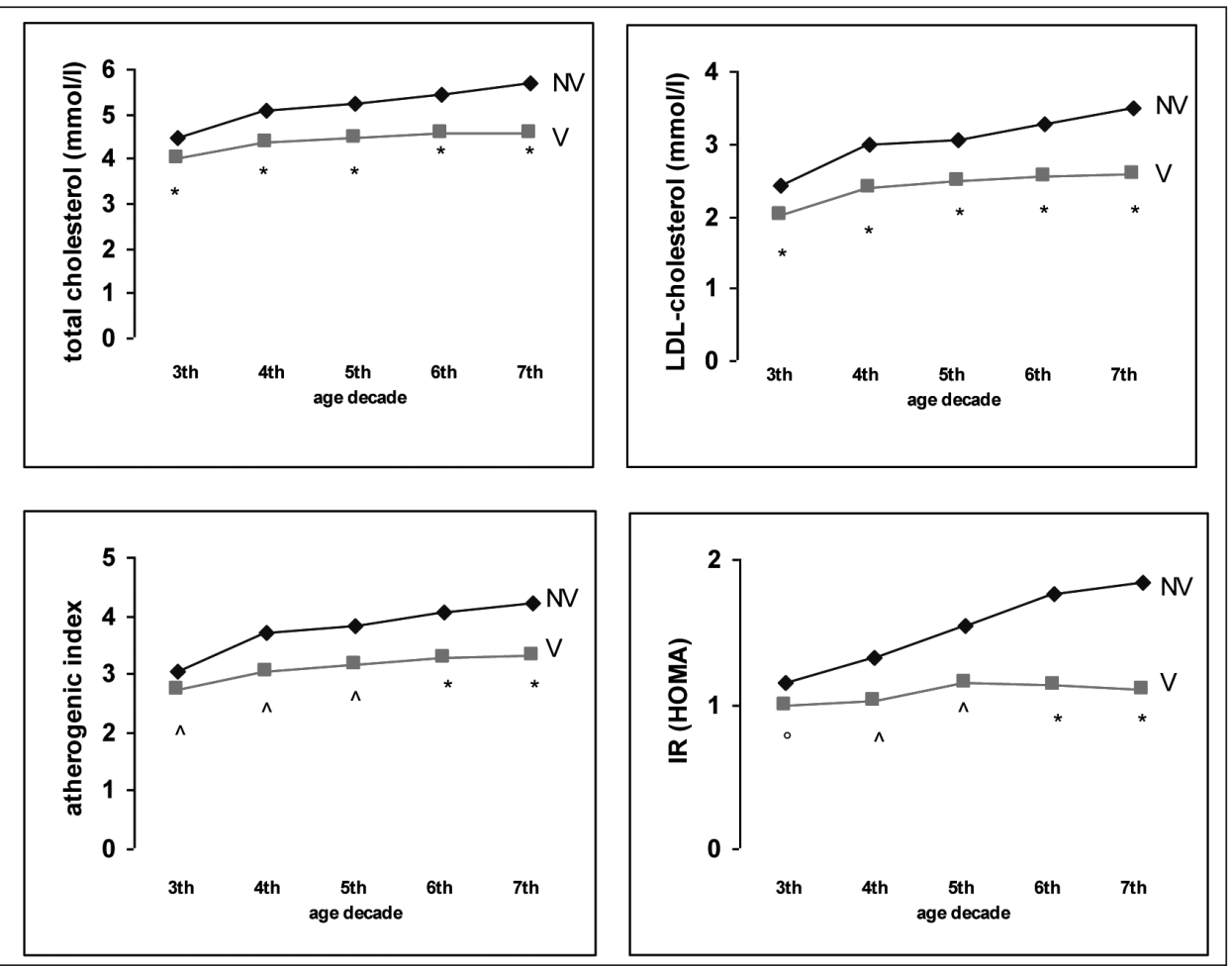

Fig.1. Total cholesterol, LDL-cholesterol, atherogenic index, IR(HOMA) values in vegetarians (V) and non-vegetarians (NV) according to age decades $\left({ }^{\circ} p<0.05,{ }^{\wedge} p<0.01,{ }^{*} p<0.001\right)$ 
etarians (148 men, 269 women; lacto-, lacto-ovo- and vegans). The mean total cholesterol and non-HDL-cholesterol concentration and the total HDL-cholesterol ratio showed expected age dependence with maximum values within the decade 60-70 years. Vegetarians showed lower total and non-HDL cholesterol concentration compared to the general population. Furthermore, the age dependent increase of these parameters was less pronounced in vegetarians. The calculated linear equations of lipid markers in dependence to age presented in our study showed that age dependent changes are also less pronounced in vegetarians (linear equations in vegetarians: total cholesterol $=0.0135$ age $+3.8036, \mathrm{r}=0.302$; LDL-cholesterol $=0.0136$ age +1.7983 , $\mathrm{r}=0.321$; atherogenic index $=0.0125$ age $+2.5001, \mathrm{r}=0.238$; linear equations in non-vegetarians: total cholesterol $=0.0303$ age $+3.8234, \mathrm{r}=0.472 ;$ LDL-cholesterol $=0.0276$ age +1.8268 , $\mathrm{r}=0.455$; atherogenic index $=0.0290$ age $+2.4651, \mathrm{r}=0.351$ ).

Cardiovascular risk can be decreased by plant protein consumption. Experimental studies described that animal proteins with higher content of essential amino acids in comparison to plant proteins induce an elevation of plasma total and LDL-cholesterol concentrations that can be prevented by a plant protein consumption $(7,16)$. Composition of dietary proteins has the potential to influence the balance of glucagon and insulin activity (17). Plant proteins are higher in non-essential amino acids compared to reference protein and other animal proteins (8). Essential amino acids are relatively more effective for releasing insulin, whereas non-essential amino acids (arginine and pyruvigenic amino acids) are effective in glucagon secretion. The effect of a chronic increase in glucagon activity by regular and sufficient intake of plant proteins means a reduction in lipogenesis, cholesterol and triacylglycerol synthesis. The higher intake of methionine and lysine from animal protein has an unfavourable effect on phospholipid metabolism (8). Subjects consuming predominantly plant food may be at lower risk of type 2 diabetes occurrence than persons on traditional mixed diet (18). Complex carbohydrates with low glycemic index are slowly absorbed and thus they have a beneficial effect on glucose control, hyperinsulinemia, insulin resistance and blood lipids. In previous study we described that vegetarians consume significantly more whole grain products, legumes, barley, oat, fruit and vegetables, which contain complex carbohydrates with soluble fiber (19).

\section{CONCLUSION}

The favourable mean age decade values of lipid and non-lipid cardiovascular risk markers in adult vegetarians aged $21-70$ years in all five age decades were significantly reduced with smaller changes between decades in comparison to non-vegetarians, thus they document a protective effect of vegetarian nutrition in prevention of cardiovascular disease. Personal clinical studies can be recommended for therapeutic nutrition of individuals with hyperlipoproteinema, DM 2 type, obesity, non alcoholic steatosis hepatis and hyperuricemic syndrome.

\section{Acknowledgement}

This publication was part of the research project "Health effects of plant food and the possibility of reduction of health risks", ITMS code: 26240220022.

\section{Conflict of Interests}

None declared

\section{REFERENCES}

1. Vasto S, Scapagnini G, Bulati M, Candore G, Castiglia L, ColonnaRomano G, et al. Biomarkers of aging. Front Biosci (Schol Ed). 2010 Jan 1;2:392-402.

2. Simm A, Nass N, Bartling B, Hofmann B, Silber RE, Navarrete Santos A. Potential biomarkers of ageing. Biol Chem. 2008 Mar;389(3):257-65.

3. Ramic E, Pranjic N, Batic-Majunovic O, Karic E, Alibasic E, Alic A. The effect of loneliness on malnutrition in elderly population. Med Arh. 2011;65(2):92-5.

4. Graham I, Atar D, Borsch-Johnsen K, Boysen G, Burell G, Cifkova R, et al; co-experts. European guidelines on cardiovascular disease prevention in clinical practice: full text. Fourth Joint Task Force of the European Society of Cardiology and other societies on cardiovascular disease prevention in clinical practice (constituted by representatives of nine societies and by invited experts). Eur J Cardiovasc Prev Rehabil. 2007 Sep;14 Suppl 2:S1-113.

5. Chaudhry KN, Chavez P, Gasowski J, Grodzicki T, Messerli FH. Hypertension in the elderly: some practical considerations. Cleve Clin J Med. 2012 Oct;79(10):694-704

6. Ginter E, Šimko V, Wsolová L. Fall of the iron curtain: male life expectancy in Slovakia, in the Czech Republic and in Europe. Cent Eur J Public Health. 2009 Dec;17(4):171-4.

7. Carroll KK, Kurowska EM. Soy consumption and cholesterol reduction, review of animal and human studies. J Nutr. 1995 Mar;125(3 Suppl):594S$597 \mathrm{~S}$.

8. Krajcovicova-Kudlackova M, Babinska K, Valachovicova M. Health benefits and risks of plant proteins. Bratisl Lek Listy. 2005;106(6-7):231-4.

9. Key TJ, Appleby PN, Rosell MS. Health effects of vegetarian and vegan diets. Proc Nutr Soc. 2006 Feb;65(1):35-41.

10. Erkkilä A, de Mello VD, Risérus U, Laaksonen DE. Dietary fatty acids and cardiovascular disease, an epidemiological approach. Prog Lipid Res. 2008 May;47(3):172-87.

11. Krajčovičová-Kudláčková M, Valachovičová M, Pauková V, Dušinská M. Effect of diet and age on oxidative damage products in healthy subjects. Physiol Res. 2008;57(4):647-51.

12. Johnson GH, Fritsche K. Effect of dietary linoleic acid on markers of inflammation in healthy persons: a systematic review of randomized controlled trials. J Acad Nutr Diet. 2012 Jul;112(7):1029-41.

13. Rajaram S, Sabaté J. Health benefits of a vegetarian diet. Nutrition. 2000 Jul-Aug;16(7-8):531-3.

14. Huang T, Yang B, Zheng J, Li G, Wahlqvist ML, Li D. Cardiovascular disease mortality and cancer incidence in vagatarians: a meta-analysis and systematic review. Ann Nutr Metab. 2012;60(4):233-40.

15. Richter V, Rassoul F, Hentschel B, Kothe K, Krobara M, Unger R, et al. Age-dependence of lipid parameters in the general population and vegetarians. Z Gerontol Geriatr. 2004 Jun;37(3):207-13.

16. McCarty MF. Vegan proteins may reduce risk of cancer, obesity, and cardiovascular disease by promoting increased glucagon activity. Med Hypotheses. 1999 Dec;53(6):459-85.

17. Tonstad S, Butler T, Yan R, Fraser GE. Type of vegetarian diet, body weight, and prevalence of type 2 diabetes. Diabetes Care. 2009 May;32(5):791-6.

18. Reaven GM. Diet and syndrome X. Curr. Curr Atheroscler Rep. 2000 Nov;2(6):503-7.

19. Valachovičová M, Krajčovičová-Kudláčková M, Blažíček P, Babinská K. No evidence of insulin resistance in normal weight vegetarians. A case control study. Eur J Nutr. 2006 Feb;45(1):52-4

Received August 19, 2015 Accepted in revised form April 17, 2017 